\title{
Infección por Leuconostoc en pacientes con síndrome de intestino corto, nutrición parenteral y alimentación enteral continua
} Leonor Jofré M., Andrea Sakurada Z., M. Teresa Ulloa F., J. Carlos Hormázabal O., Viviana Godoy M.,
Jorge Fernández O., Marcela Gutiérrez M., M. Pilar Monteverde O., Marcela Castillo G. y Ana Canales P.

\section{Hospital Clínico Universidad de Chile Servicio de Pediatría (LM, MGM, MPMO) Laboratorio de} Microbiología (ASZ, MCG, ACP)

Facultad de Medicina Universidad de Chile, Instituto de Ciencias Biomédicas Programa de Microbiología (MTUF).

Instituto de Salud Pública de Chile (JCHO, VGM, JFO)

Recibido: 6 octubre 2005 Aceptado: 25 mayo 2006

Correspondencia a: Leonor Jofré Morales leonorjofre@gmail.com

\section{Leuconostoc infcctions in patients with short gut syndrome, parenteral nutrition and continuous enteral feeding}

Leuconostoc is a grampositive cocci, quite ubiquitous in nature. It is used in wine industry, and for aroma and texture of dairy products. Occasionally it has been isolated from humans in cases of bacteremia, catheter associated infections, sepsis, meningitis, pneumonia, UTI, osteomyelitis and hepatic dysfunction. Short bowel syndrome, patients with CVC and patients with gastrostomy undergoing enteral feeding, are described amongst the factors associated with this infection. The isolation of a grampositive cocci, that does not hydrolyze arginine and that is resistant to vancomycin leads to this diagnostic possibility. Antibiotic treatment: penicillin or ampicillin.

Key words: Leuconostoc; bacteremia; parenteral nutrition; enteral feeding; short gut syndrome; Leuconostoc garlicum.

Palabras claves: Leuconostoc; bacteriemia; nutrición parenteral; alimentación enteral; síndrome de intestino corto; Leuconostoc garlicum.

\section{Introducción}

$L$ euconostoc es una bacteria cocácea grampositiva catalasa negativa que induce un primer diagnóstico hacia el género Streptococcus. Tiene una amplia distribución en la naturaleza, encontrándose en vegetales verdes y raíces, que son su nicho ecológico natural. Es usada en la producción de vinos, quesos, productos lácteos y azúcares, donde juega un rol esencial en la mezcla de aromas y texturas. Se encuentra en la microbiota de vacunos, insectos y en residuos trata$\operatorname{dos}^{1}$. No forma parte de la microbiota del hombre, ocasionalmente se ha aislado de deposiciones, secreción vaginal y leche materna, y se asocia esporádicamente a infección. Una característica trascendente de Leuconostoc es su resistencia a vancomicina ${ }^{2}$. La infección por este agente se ha descrito en pacientes con patologías gastrointestinales, catéter venoso central (CVC), nutrición parenteral (NP) o alimentación enteral continua (AEC).

Presentamos 2 casos de lactantes con síndrome de intestino corto, manejadas con NP crónica y AEC que presentaron cultivos positivos a Leuconostoc, grupo de particular riesgo para adquirir infección por este agente ${ }^{3,4}$.

\section{Caso 1}

Lactante de 12 meses, sexo femenino, con antecedentes de haber sido RNTAEG y síndrome de Apple Peel, operada en 3 oportunidades por obstrucción intestinal en el período de RN. Se le realizó resección intestinal quedando con $29 \mathrm{~cm}$ de intestino delgado y válvula ileocecal. Fue tratada con NP a través de un CVC tipo Broviac y con AEC a bajos volúmenes como estímulo enteral por gastrostomía con sonda yeyunal.

Durante los primeros meses de vida presentó cuadros infecciosos intercurrentes: una infección asociada a catéter por Staphylococcus aureus resistente a oxacilina, tratada con vancomicina durante 14 días; diarrea por Escherichia coli enteropatógena (ECEP) y rotavirus (RTV) e infecciones respiratorias altas de etiología viral.

Al año de vida presentó un cuadro de diarrea, con fiebre, decaimiento e intolerancia a la $\mathrm{AEC}$, los exámenes solicitados fueron sugerentes de una infección bacteriana con PCR de $93 \mathrm{mg} / \mathrm{lt}$ (VN $<$ de $10 \mathrm{mg} / \mathrm{lt}$ ) y en el hemograma una leucocitosis de $15.000 / \mathrm{mm}^{3}$, sin desviación a izquierda ni neutrofilia. El coprocultivo fue positivo a ECEP, con leucocitos fecales (LF) negativos y RTV (-), por lo que se indicó tratamiento con neomicina. 
Presentó un urocultivo positivo a Klebsiella pneumoniae, con un sedimento de orina no sugerente de infección pero, por continuar febril, se decidió indicar cefotaxima; el urocultivo repetido fue negativo. Por persistencia de la curva febril se asoció vancomicina. Dos hemocultivos periféricos tomados al inicio del cuadro fueron informados positivos a una cocácea grampositiva en estudio, posible Streptococcus, con resistencia a vancomicina y cefotaxima, de acuerdo a las tablas NCCLS para Streptococcus no pneumoniae ${ }^{5}$. La cepa fue enviada al ISP, laboratorio de referencia nacional, para su identificación. El hemocultivo cuantitativo tomado por catéter fue informado como negativo.

Durante la evolución del cuadro febril, el catéter Broviac se fracturó siendo reemplazado por uno nuevo y la sonda de yeyunostomía se cambió al desplazarse la anterior. Las deposiciones continuaron alteradas, con distensión abdominal e intolerancia al reinicio de la AEC; con la sospecha de infección abdominal se agregó metronidazol. De allí en adelante disminuyeron la curva febril y frecuencia de deposiciones, mejorando los parámetros de laboratorio. Completó 14 días de tratamiento con vancomicina, cefotaxima y 7 días de metronidazol, con buena evolución.

Posteriormente se recibió el informe del ISP que identificaba la cepa enviada a estudio como Leuconostoc sp. La confirmación del ISP se realizó de acuerdo a las características microbiológicas de la cepa (Tabla 1), y como parte de un protocolo de investigación, se realizó la amplificación y secuenciación automática del fragmento $16 \mathrm{~S}$ rARN. Las secuencias obtenidas fueron analizadas y reconocidas en la base de datos GeneBank como Leuconostoc garlicum. En cuadros infecciosos posteriores no se volvió a aislar este agente.

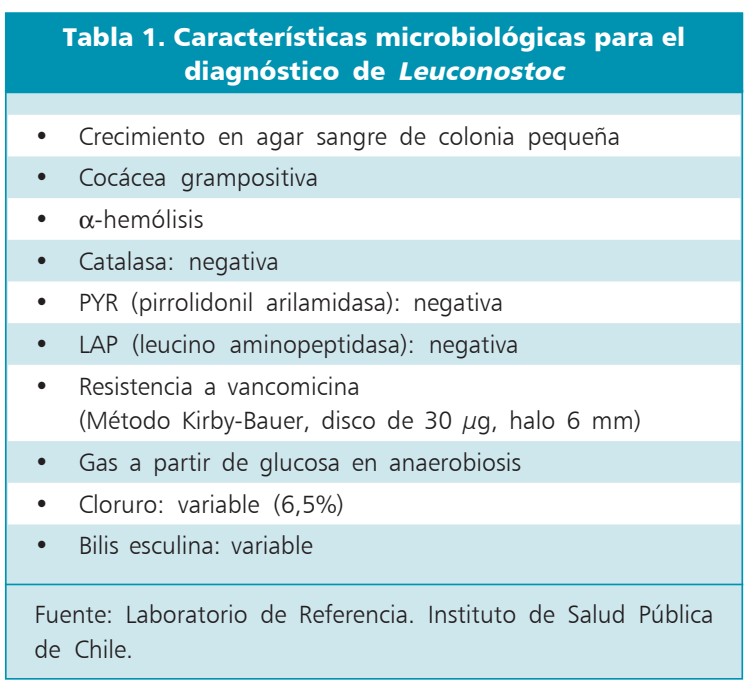

\section{Caso 2}

Lactante de 8 meses, sexo femenino, antecedente de haber sido RNTAEG. A los 3 días de vida presentó un cuadro compatible con obstrucción intestinal, realizándosele una resección intestinal e ileostomía; el estudio histológico de la pieza operatoria fue compatible con una enfermedad de Hirschprung pancolónica. Por nuevos cuadros de obstrucción fue necesario resecar parte del intestino delgado y la válvula ileocecal, quedando con $27 \mathrm{~cm}$ de intestino y un síndrome de intestino corto funcional; requirió aporte de NP desde el segundo día de vida y AEC en volúmenes progresivos con leche materna, hasta casi llegar al aporte total el primer mes. A los 2 meses se le instaló CVC central tipo Broviac.

Durante su estadía presentó una infección asociada a catéter por $S$. aureus resistente a oxacilina, tratada con vancomicina durante 14 días, al cuarto mes de vida una diarrea por RTV y enterocolitis necrosante (ECN) tratada con vancomicina, cefotaxima y metronidazol, al quinto mes presentó un nuevo episodio de diarrea, tratada con metronidazol y ciprofloxacina endovenosos durante 10 días, con LF $(+++)$, coprocultivo negativo y estudio con ELISA para RTV (-). Al $6^{\circ}$ mes otro episodio de infección asociada a catéter por Staphylococcus coagulasa negativa, tratada con vancomicina y retiro del catéter, por persistencia de la fiebre. Se le realizó un estudio inmunológico encontrándose una alergia a la proteína de leche de vaca, ADN y pollo, por lo que se

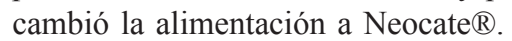

A los 8 meses inició un nuevo cuadro febril, con dolor y eritema de la región perigastrostomía, se descartó una complicación quirúrgica y se inició tratamiento con cloxacilina y amikacina. Se mantuvo febril, con pérdidas aumentadas por ileostomía, la ecografía abdominal mostró asas intestinales de pared algo engrosada y la ecocardiografía no mostró vegetaciones; completó 10 días de tratamiento antimicrobiano.

Dos semanas después inició un cuadro séptico por lo que se trasladó a la Unidad de Cuidados Intensivos Pediátricos (UCIP). Entre los exámenes solicitados destacó una PCR de $78 \mathrm{mg} / \mathrm{lt}$ (previa de $17 \mathrm{mg} / \mathrm{lt}$ ), hemograma con hematocrito $26 \%, \mathrm{Hb} 8 \mathrm{gr} / \mathrm{dl}$, leucocitos $7.000 / \mathrm{mm}^{3}$ (segmentados $74 \%$, baciliformes $1 \%$ ) y VHS de $56 \mathrm{~mm} / \mathrm{h}$. Fue tratada con aporte de volumen, sin necesidad de apoyo con aminas vasoactivas, y vancomicina.

Los hemocultivos fueron informados positivos a una cocácea grampositiva en estudio, posible Streptococcus y se agregó al esquema amikacina. El estudio de susceptibilidad de la cepa, usando las tablas para Streptococcus no pneumoniae de la NCCLS ${ }^{5}$, mostró resistencia a vancomicina, cefotaxima y sensibilidad 
intermedia a penicilina. La paciente fue tratada con clindamicina y amikacina.

Mantuvo alzas térmicas hasta el cuarto día de tratamiento decidiéndose el retiro del catéter Broviac y su reemplazo por un catéter femoral; con ello cayó la curva febril. El cultivo de la punta de catéter fue informado como positivo a $S$. aureus resistente a oxacilina, recibiendo 12 días de clindamicina y amikacina con buena evolución.

Se informó posteriormente que la cocácea grampositiva aislada de hemocultivos, estudiada con sistema API 20 Strep Bio Merieux, Inc. correspondía a Leuconostoc sp: VP (+), Esc (+), PYR (-), $\alpha$-GAL 55\%, $\beta$-GAL (+), LAP (-) y tetralosa (+). En este caso no se realizó estudio molecular por no disponer de la cepa.

Con posterioridad a este cuadro presentó un tromboembolismo pulmonar que se trató con heparina de bajo peso molecular por tiempo prolongado y reinició la AEC hasta llegar al volumen total. Se dio de alta en buenas condiciones.

\section{Discusión}

Leuconostoc deriva de la palabra latina leucus (= claro o incoloro) y nostoc, que es el nombre genérico de alga. Este género pertenece al grupo de bacterias productoras de ácido láctico y se encuentran en el medio ambiente, en materia vegetal, productos lácteos y vinos. Son cocáceas grampositivas, no esporuladas, agrupadas en pares o cadenas, que parecen Streptococcus, aerobios facultativos, catalasa negativa, con resistencia intrínseca a vancomicina ${ }^{1,2,6}$.

Estudios con secuenciación de los fragmentos $16 \mathrm{~S}$ rARN y 23S ADN la agrupan en tres lineajes: género Leuconostoc "sensu stricto", Weisella y Oenococcus. El género Leuconostoc comprende varias subespecies, entre las que destacan: L. mesenteroides, L. dextranicum, L. cremori. y L. citreum. El género Weissella incluye a $W$. viridescens y otros cuatro lactobacilos hetero-fermentadores. El grupo Oenococcus está representado por $O$. oeni ${ }^{7}$ (Tabla 2).

Leuconostoc se desarrolla bien en placas de agar sangre (Figura 1), es de forma esférica o lenticular y mide de 0,5 a $0,7 \mu$ de ancho por 0,7 a $1 \mu$ de longitud. La temperatura óptima para su desarrollo es entre 20 y $30^{\circ}$ $\mathrm{C}$, con un $\mathrm{pH}$ entre 4,4 y 5. En la superficie del agar son $\alpha$-hemolíticas o no hemolítica, por lo que puede confundirse con otros Streptococcus grupo viridans razón por la que el laboratorio evaluó resistencia a vancomicina por difusión-, Enterococcus o Lactococcus sp. El 30\% de estas bacterias pueden reaccionar con antisueros contra grupo $\mathrm{D}^{2,8}$.

La identificación se realiza en base a las siguientes características: cocácea grampositiva, catalasa negativa, PYR (pirrolidonil arilamidasa) y LAP (leucino aminopeptidasa) negativas y resistencia a vancomicina en disco de $30 \mu \mathrm{g}$ (Figura 2) ${ }^{9,10}$. Producen $\mathrm{CO}_{2}$ a partir de la glucosa, no hidroliza la arginina,

La resistencia a vancomicina es de tipo cromosomal y cruzada con teicoplanina. Leuconostoc posee una enzima penta depsipéptido ligasa LmDd12, que tiene un carbono $\mathrm{C}$ terminal D-lactato en vez de D-alanina, genera un péptido intermediario terminado en Dalanina-D-lactato, que es de menor afinidad a glicopéptidos $^{11}$.

Presenta sensibilidad intermedia a penicilina, ampicilina, clindamicina, eritromicina y aminoglucósidos y sensibilidad intermedia a cefalosporinas e imipenen. Se han descrito cepas resistentes a cotrimoxazol y sulfonamidas ${ }^{2,12}$. Leuconostoc no es una especie detectada con frecuencia, en el ISP desde el año 2003 a la fecha se ha confirmado en 5 cepas aisladas de hemocultivo. No encontramos referencias nacionales publicadas en relación a infecciones por este agente.

Es una bacteria oportunista por afectar con mayor frecuencia a pacientes inmunocomprometidos y $\mathrm{RN}^{13}$. El $60 \%$ de los casos descritos se presenta en pacientes pediátricos con patologías gastrointestinales y sín-

\section{Tabla 2. Genéro Leuconostoc}

\section{Género}

Leuconostoc

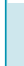

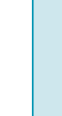


drome de intestino corto. Se ha descrito un tercio de los casos en $\mathrm{RN}$ prematuros y $11 \%$ en pacientes con alteración de la inmunidad, como es el caso de quienes se someten a trasplantes de órganos, pacientes oncológicos o con síndrome de inmunodeficiencia adquiri$\mathrm{da}^{13-17}$. El uso de CVC es un factor de riesgo presente en la mitad de los casos, al igual que el uso previo de vancomicina $^{12}$. En pacientes adultos se asocia a insuficiencia hepática crónica, insuficiencia renal crónica con hemodiálisis o peritoneo-diálisis y lupus eritematoso sistémico ${ }^{13}$, que presentan un cuadro de mayor gravedad. Se ha descrito bacteriemia en un gran quemado, portador de CVC y con AEC ${ }^{18}$.

En infecciones humanas se ha aislado L. mesenteroides, L. pseudomesenteroides, L. citreum y L. lactis ${ }^{2}$, puede encontrarse en procesos polimicrobianos ${ }^{19} \mathrm{y}$ en un pequeño porcentaje, en la microbiota intestinal de los $\mathrm{RN}^{20}$. Se ha podido aislar de hemocultivos, líquido pleural, LCR, líquido ascítico, orina, tejido óseo y en muestras obtenidas por lavado broncoalveolar 14,21-25. Puede producir meningitis ${ }^{26,27}$, ventriculitis ${ }^{28}$, peritonitis $^{29}$, abscesos hepáticos ${ }^{30}$, abscesos $\operatorname{mamarios}^{31}$, infección de origen dental ${ }^{32}$, infección asociada a catéter $^{23}$, osteomielitis ${ }^{33,34}$, endoftalmitis ${ }^{35}$, neumonía ${ }^{14}$, endocarditis $^{36}$, bacteriemia ${ }^{37}$ y síndromes sépticos ${ }^{3}$. Está descrito un brote nosocomial por este agente en pacientes críticos $^{38}$ y casos de infección urinaria asociada a catéter urinario permanente ${ }^{39,40}$. No se ha descrito mortalidad asociada a infección por este agente ${ }^{23}$.

En los pacientes con síndrome de intestino corto, que requieren de NP y AEC, se postula una vía de entrada gastrointestinal por contaminación de las fórmulas enterales, debido a que es posible encontrar a Leuconostoc en los productos lácteos y en los mezcladores usados en la preparación de fórmulas ${ }^{4,13}$. En

Tabla 3. Características que permiten

la identificación de Leuconostoc en Laboratorios de Microbiología *

- Cocácea gram positiva en cadena parecida a Streptococcus

- $\alpha$-hemólisis

- Catalasa: negativa

- PYR: negativa

- LAP: negativa

- Resistencia a vancomicina

- Bilis esculina: variable

- Crecimiento en $\mathrm{NaCl} 6,5 \%$

* Adaptado de referencias $2,7,9,40$

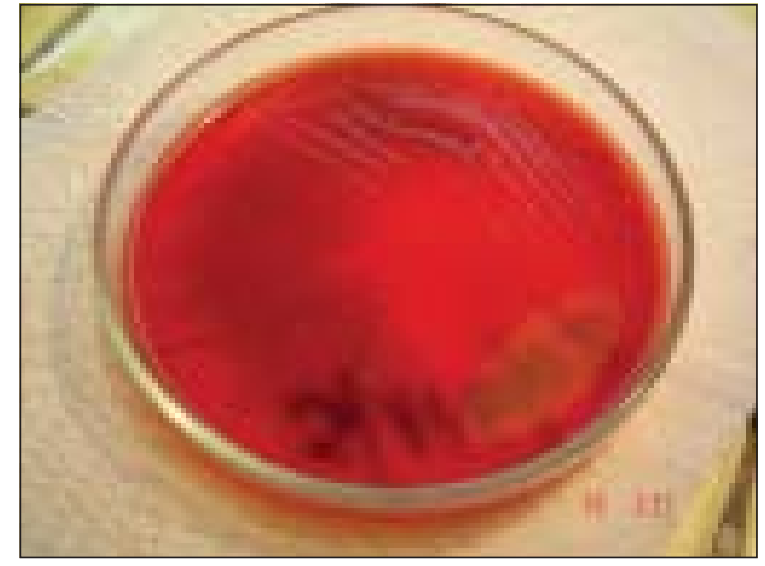

Figura 1. Aspecto de la coIonia de Leuconostoc en agar sangre.

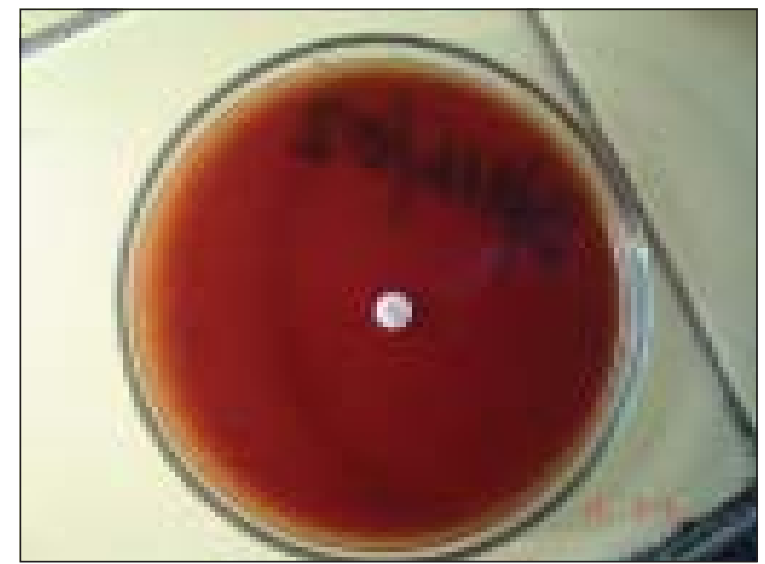

Figura 2. Estudio de susceptibilidad en Leuconostoc sp a vancomicina muestra la ausencia de halo de inhibición.

ninguno de nuestros casos fue posible realizar este estudio porque al momento de la identificación, los tarros de las fórmulas y las bajadas se habían eliminado. Se debe considerar la diseminación por vía hematógena, a través del CVC, o por sondas de alimentación que producen microlesiones en el tracto gastrointestinal, facilitando la entrada hacia el sistema circulatorio. En el caso de una infección asociada a catéter, el retiro ayuda a controlar la infección, lo que ocurrió en el caso 1 en que se cambió el catéter Broviac por fractura del dispositivo además del cambio de la sonda yeyunal, que podría haber representado otra puerta de entrada.

Con el aumento de la población inmunocomprometida, el uso de CVC de larga duración, NP, AEC $\mathrm{y}$ tratamientos previos con vancomicina, la presencia de estos géneros resistentes a vancomicina como Leuconostoc y Pediococcus, debe ser considerada. El informe al médico tratante de una cocácea grampositiva, catalasa negativa, resistente a vancomicina, será de 
mayor utilidad que informar Streptococcus en estudio $^{41}$. El diagnostico de género, ya sea mediante pruebas convencionales o sistema comercial como el API, podría realizarse en cualquier laboratorio de Microbiología, de lo contrario, la cepa debiera ser enviada al laboratorio de referencia. Desde el punto de vista clínico, la determinación de género es suficiente, dado que la identificación de especie no modificará la conducta terapéutica. La determinación por secuenciación del gen $16 \mathrm{~S}$ rARN de L. garlicum se realiza por primera vez en nuestro medio; esta especie fue descrita recientemente y no se ha reportado asociación con infección en el hombre.

El estudio de susceptibilidad no está estandarizado, en caso de realizarlo debe ser hecho mediante $\mathrm{CIM}^{42}$. Los antimicrobianos de elección en infecciones por Leuconostoc son penicilina y ampicilina, en cuadros graves puede asociarse clindamicina ${ }^{2,12}$.

\section{Resumen}

Leuconostoc es una cocácea grampositiva parecida a los Streptococcus, que se encuentra ampliamente distribuida en la naturaleza; es usada en la industria de vinos, productos lácteos y quesos para la producción de aromas y texturas. Leuconostoc causa ocasionalmente infecciones en humanos, puede producir bacteriemia, infección asociada a catéter, síndrome séptico, meningitis, neumonía, infección del tracto urinario, osteomielitis y compromiso hepático, entre otros. Se describen como factores de riesgo para una infección por este agente: el síndrome de intestino corto, uso de catéter venoso central y la alimentación enteral por gastrostomía. Orientan a la presencia de este agente el aislamiento de una cocácea grampositiva, catalasa negativa, PYR y LAP negativas, resistente a vancomicina. El tratamiento de elección es penicilina o ampicilina.

\section{Referencias}

1.- Hemme D, Foucaud-Scheunemann C. Leuconostoc, characteristics, use in diary technology and prospects in functional foods. Int Dairy J 2004; 14: 467-94.

2.- Facklam R, Elliot J A. Identification, classification and clinical relevance of catalase-negative, gram positive cocci, excluding the Streptococci and Enterococci. Clin Microbiol Rev 1995; 8: 479-95.

3.- Monsean T, Granlund M, Olofsson K, Olsen B. Leuconostoc spp. septicaemia in a child with short bowel syndrome. Scand J Infect Dis 1997; 29: 310-1.

4.- Dhodapkar K M, Henry N. Leuconostoc bacteremia in an infant with short-gut syndrome: case report and literature review. Mayo Clin Proc 1996; 71: 1171-4.

5.- Swenson J M, Facklam R R, Thornsberry C. Antimicrobial susceptibility of vancomycinresistant Leuconostoc, Pediococcus and Lactobacillus species. Antimicrob Agents Chemother 1990; 34: 543-9.

6.- Ruoff K L. Miscellaneous catalase-negative, gram positive cocci: emerging opportunists. J Clin Microbiol 2002; 40: 1129-33.

7.- Jang J, Kim B, Lee J, Han H. A rapid method for identification of typical Leuconostoc species by $16 \mathrm{~S}$ rDNA PCR-RFLP analysis. J Microbiol Methods 2003; 55: 295-302.

8.- Isenberg H D, Vellozi E M, Shapiro J, Rubin L G. Clinical laboratory in the recognition of Leuconostoc spp. J Clin Microbiol 1988; 26: 479-83.

9.- Facklam R, Pigott N, Franklin R, Elliot J. Evaluation of three disk test for identification of Enterococci, Leuconostoc and Pediococci. J Clin Microbiol 1995; 33: 885-7.

10.- Ruoff K L, Kuritzkes D R, Wolfson J S, Ferraro M J. Vancomycin resistant Gram positive bacteria isolated from human sources. J Clin Microbiol 1988; 26: 2064- 8.

11.- Kuzin A P, Sun T, Jorczak-Baillass J, Healy V, Walsh C, Knox J R. Enzymes of vancomycin resistance: the structure of $\mathrm{D}$ alanine-D-lactate ligase of naturally resistant Leuconostoc mesenteroides. Structure 2000; 8: 463-70.

12.- Swenson J M, Facklam R R, Thornsberry C. Antimicrobial susceptibility of vancomycinresistant Leuconostoc, Pediococcus and Lactobacillus species. Antimicrob Agents Chemother 1990; 34: 543-9.

13.- Carapetis J, Bishop S, Davis J, Bell B, Hogg G. Leuconostoc sepsis in associated with continuous enteral feeding: two case reports and review. Pediatr Infect Dis J 1994; 13: 816- 23.

14.- Giacometti A, Ranaldi R, Siquini F M, Scalise G. Leuconostoc citreum isolated from lung in AIDS patient. Lancet 1993; 342: 622 .

15.- Casanova Román M, Ríos J, Sánchez-Porto A, Gomar J L, CasanovaBellido M. Leuconostoc bacteremia in a healthy infant. Minerva Pediatr 2003; 55: 83-6.

16.- Ferrer S, de Miguel G, Domingo P, Pericas R, Prats G. Pulmonary infection due to Leuconostoc species in a patient with AIDS. Clin Infect Dis 1995; 21: 225-6.

17.- Zinner S. Changing epidemiology of infections in patients with neutropenia and cancer: Emphasis on Gram positive and resistant bacteria. Clin Infect Dis 1999; 29: 490-4.

18.- Jiménez- Mejías M E, Becerril B, Gómez-Cia T, Del Nozal M, PalominoNicas J. Bacteremia caused by Leuconostoc cremoris in a patient with severe burn injuries. Eur J Clin Microbiol Infect Dis 1997; 16: $533-5$.

19.- Reyna-Figueroa J, Ramírez- Landin A, Maldonado J G, Ortiz-Ibarra F J. Sepsis neonatal polimicrobiana por Leuconostoc sp y Candida parapsilosis. Reporte de un caso en el Instituto Nacional de Perinatología. Rev Enfer Inf Ped 2005; 73: 30-3.

20.- Hee-Kyung P, Sung-Suh S, Su-Yung K, Jae-Hong P, Su-Eun P, Hak-Jung K et al. Molecular analysis of colonized bacteria in a human newborn infant gut. J Microbiol 2005; 43: 345-53

21.- Giraud P, Aytal M, Lemouzy J, Huguet F, Schlaifer F, Pris J. Leuconostoc, a potential pathogen in bone marrow transplantation. Lancet 1995; 341: 1481-2.

22.- Jato A, García- Zabarte ML, Jato M L, Varela E, García-Riestra C, Regueiro B. Bacteriemia en una niña con rabdomiosarcoma. Enferm Infecc Microbiol Clin 2002; 20: 85-7.

23.- Hardy S, Ruoff K L, Catlin E, Santos J I. Catheter associated infection with a vancomycin resistant gram positive coccus of the Leuconostoc sp. Pediatr Infect Dis J 1988; 7: 519-20.

24.- Fauchis A L, Roques S, Druesne L, Verdonck A, Chassagne P, Bercoff E. Uncommon opportunist infection due to Leuconostoc special. Rev Med Interne 2003; 24: $268-75$. 
25.- Borer A, Weber G, Avnon L S, Riesenberg K, Alkan M. Pleural empyema caused by Leuconostoc spp. Scand J Infect Dis 1997; 29: 311-2.

26.- Friedland I R, Snipelisky M, Khoosal M. Meningitis in a neonate caused by Leuconostoc sp. J Clin Microbiol 1990; 28: 2125-6.

27.- Coodavia Y M, Solwa Z, van den Ende J. Meningitis caused by vancomycin-resistant Leuconostoc sp. J Clin Microbiol 1987; 25: 1784-5.

28.- Deye G, Lewis J, Patterson J, Jorgensen J. A case of Leuconostoc ventriculitis with resistance to carbapenem antibiotics. Clin Infect Dis 2003; 37: 869-70.

29.- Gillespie R, Symons J M, McDonald R A. Peritonitis due to Leuconostoc species in a child receiving peritoneal dialysis. Pediatr Nephrol 2002; 17: 966-8.

30.- Montejo M, Grande C, Valdivieso A, Testillano M, Minguillan J, Aguirrebengoa K, et al. Abdominal abscess due to Leuconostoc species in a liver transplant recipient. J Infect 2000; 41: 197-8.

31.- Barry H, Clancy M T, Brady A,
O'Higgins N. Isolation of a Leuconostoc species in a patient from retroareolar breast abscess. J Infect 1993; 27: 208-10.

32.- Wenocur H S, Smith M A, Vellozzi E M, Shapiro J, Isenberg H. Odontogenic infection secondary to Leuconostoc species. J Clin Microbiol 1988; 26: 1893-4.

33.- Zaoui A, Brousse C, Bletry O, Augouard L W, Boisaubert B. Leuconostoc osteomyelitis. Joint Bone Spine 2005; 72: 79-81.

34.- Mulford J S, Mills J. Osteomyelitis caused by Leuconostoc species. Austr NZ J Surg 1999; 69: $541-2$.

35.- Kumudhan D, Mars S. Leuconostoc mesenteroides as a cause of post operative endophtalmitis- a case report. Eye 2004; 18: 1023-4.

36.- Vázquez E, Carazo I, Martín A, Lozano C, Cuesta I, Pagola C. Endocarditis infecciosa por Leuconostoc mesenteroides. Enferm Infecc Microbiol Clin 1998; 16: 237-8.

37.- Goenaga M A, Alberdi F, Carrera J A, Millet M, Garde Orbaiz C. Bacteriemia por Leuconostoc spp en un paciente con síndrome de pseudoobstrucción intestinal. An Med Interna 2003; 20: 53- 4.
38.- Scano F, Rosi L, Catteland A, Carretta G, Meneghetti F, Cadrobbi P, et al. Leuconostoc species: a case cluster hospital infection. Scand J Infect Dis 1999; 31: 371-3.

39. - Cappelli E A, Barros R R, Camello T C, Teixieira L M, Merquior V L. Leuconostoc pseudomesenteroides as a cause of nosocomial urinary tract infections. J Clin Microbiol.1999; 37: 4124-6.

40.- Taneja N, Rani P, Emmanuel R, Khudaier B Y, Sharma S K, Sharma M. Nosocomial urinary tract infection due to Leuconostoc mesenteroides at a tertiary care center in north India. Indian J Med Res 2005; 122: 178-9.

41.- Palavecino E. Streptococcus anginosus: ¿Es su identificación clínicamente importante?. Rev Chil Infect 2004; 21: 261-7.

42.- Ruoff R K. Chapter 31: Aerococcus, Abiotrophia, and other infrequently isolated aerobic, catalase (-) grampositive cocci. Murray R. Baron, Jorgensen M, Pfaller M, Yolken R. Manual of Clinical Microbiology. $20038^{\text {th }}$ Ed. ASM Press Washington DC, pág 434-44. 